\title{
Prospective ECG triggering reduces prosthetic heart valve-induced artefacts compared with retrospective ECG gating on 256-slice CT
}

\author{
Petr Symersky • Jesse Habets • Paul Westers • \\ Bas A. J. M. de Mol • Mathias Prokop • \\ Ricardo P. J. Budde
}

Received: 24 July 2011 /Revised: 20 November 2011 / Accepted: 22 November 2011 /Published online: 30 December 2011

(C) The Author(s) 2011. This article is published with open access at Springerlink.com

\begin{abstract}
Objectives Multidetector computed tomography (MDCT) has diagnostic value for the evaluation of prosthetic heart valve (PHV) dysfunction but it is hampered by artefacts. We hypothesised that image acquisition using prospective triggering instead of retrospective gating would reduce artefacts related to pulsating PHV.

Methods In a pulsatile in vitro model, a mono- and bileaflet PHV were imaged using $256 \mathrm{MDCT}$ at 60, 75 and 90 beats per minute (BPM) with either retrospective gating $(120 \mathrm{kV}$, $600 \mathrm{mAs}$, pitch $0.2, \mathrm{CTDI}_{\mathrm{vol}} 39.8 \mathrm{mGy}$ ) or prospective triggering $\left(120 \mathrm{kV}, 200 \mathrm{mAs}, \mathrm{CTDI}_{\mathrm{vol}} 13.3 \mathrm{mGy}\right)$. Two thresholds ( $>175$ and $<-45 \mathrm{HU})$, derived from the density
\end{abstract}

P. Symersky $(\bowtie) \cdot$ B. A. J. M. de Mol

Department of Cardiothoracic Surgery, Academic Medical Center,

Meibergdreef 9 ,

1105 AZ Amsterdam, the Netherlands

e-mail: psymersky@gmail.com

J. Habets $\cdot$ R. P. J. Budde

Department of Radiology, University Medical Center Utrecht,

Utrecht, the Netherlands

P. Westers

Department of Biostatistics,

Julius Center for Health Sciences and Primary Care,

University Medical Center Utrecht,

Utrecht, the Netherlands

B. A. J. M. de Mol

Department of Biomedical Engineering,

University of Technology Eindhoven,

Eindhoven, the Netherlands

M. Prokop

Department of Radiology,

Radboud University Nijmegen Medical Center,

Nijmegen, the Netherlands of surrounding structures, were used for quantification of hyper- and hypodense artefacts. Image noise and artefacts were compared between protocols.

Results Prospective triggering reduced hyperdense artefacts for both valves at every BPM ( $P=0.001$ all comparisons). Hypodense artefacts were reduced for the monoleaflet valve at $60(P=0.009), 75(P=0.016)$ and $90 \mathrm{BPM}(P=0.001)$, and for the bileaflet valves at $60(P=0.001), 90(P=0.001)$ but not at 75 BPM $(P=0.6)$. Prospective triggering reduced image noise at $60(P=0.001)$ and $75(P<0.03)$ but not at 90 BPM.

Conclusions Compared with retrospective gating, prospective triggering reduced most artefacts related to pulsating PHV in vitro.

Key Points

- Computed tomographic images are often degraded by prosthetic heart valve-induced artefacts

- Prospective triggering reduces prosthetic heart valveinduced artefacts in vitro

- Artefact reduction at 90 beats per minute occurs without image noise reduction

- Prospective triggering may improve CT image quality of moving hyperdense structures

Keywords Computed tomography · Heart valve prosthesis . Cardiac-gated imaging techniques $\cdot$ Artefacts .

Cardiovascular model

\section{Introduction}

Electrocardiography (ECG)-gated multidetector computed tomography (MDCT) can identify the morphological substrates of prosthetic heart valve (PHV) dysfunction [1-4]. 
Echocardiography, which is the conventional method of monitoring PHV function, may not always identify the cause of dysfunction because of acoustic shadowing [4-6]. In regions obscured by acoustic shadowing, MDCT has been shown to identify obstructive masses, thrombus, vegetations and degenerated thickened leaflets of biological prostheses $[1-4,7]$. The identification of these causes of dysfunction may guide clinical management and may even form an indication for the surgical replacement of dysfunctional valves $[1,2,4,8,9]$.

These imaging results have been achieved mostly with 64-slice systems using retrospectively ECG-gated coronary $\mathrm{CT}$ angiography protocols. With retrospectively gated acquisition, the in vitro and in vivo image quality of modern mechanical PHV was found to be generally good despite variable amounts of artefacts $[1,10-12]$. The artefacts seemed to be related to the radiopaque parts of the PHV, such as the metal alloy of the prosthetic ring and the tungsten impregnated carbon leaflets, and consisted of low attenuation (hypodense) and high attenuation (hyperdense) components $[10,12]$. Because of their resemblance to metal artefacts, probably similar causal mechanisms such as scatter, photon starvation, edge effects, beam hardening and motion may play a role [13-15]. Some of these interactions can be influenced by modifying acquisition parameters, other factors (such as motion) are patient-dependent. A pragmatic approach has been the use of modified coronary protocols with retrospective gating but with increased tube currents and voltages [3, 7]. Although these reports yielded good image quality, it was at the expense of sizable radiation exposure, which may curtail the use of CT for PHV evaluation and withhold the potential advantages of the technique to all but highly selected patients. Other adaptations for enhanced PHV imaging have not been studied and no specific PHV MDCT protocols have been developed to date.

With the introduction of 256- and 320-detector systems, PHV can be imaged within one rotation using prospective ECG-triggering. Experiments with an in vitro model of coronary in-stent restenosis suggested that prospective triggering may be associated with improved image quality compared with retrospective gating [16]. In addition, prospective triggering may achieve this with a fraction of the radiation dose compared with modified coronary protocols that have been used for PHV imaging. Because PHV also appear as high density objects on CT, a similar improvement may be possible for prospectively triggered imaging of PHV. In order to test this hypothesis we compared a retrospectively gated and a prospectively triggered protocol using a 256-detector system for imaging the two most common bileaflet and tilting disc mechanical PHVs in a pulsatile in vitro model.

\section{Materials and methods}

Two mechanical PHVs, St Jude bileaflet (SJ, St Jude Medical Inc., St Paul, MN, USA, valve size $27 \mathrm{~mm}$ ) and Medtronic Hall tilting disc (MH, Medtronic Inc., Minneapolis, MN, USA, valve size $27 \mathrm{~mm}$, Fig. 1), were inserted into a pulsatile in vitro model, which was described by Symersky et al. [10]. In brief, the valves were mounted in a polymethylmethacrylate (PMMA) valve chamber (Fig. 2) that was placed in a thoracic phantom (QRM GmbH, Möhrendorf, Germany). The valve chamber was connected to a computer controlled piston pump and to an afterload reservoir. Water was used as a perfusate. The flow pulses were at a frequency of 60,75 and 90 beats per minute (BPM) and produced identical pulsation cycles. The valves were opened from 30 to $50 \%$ of the artificial ECG interval generated by the computer.

Imaging was performed using 256 MDCT (iCT, Philips Medical Systems, Best, The Netherlands). Two imaging protocols were used: 1) a standard helical retrospectively ECG-gated protocol based on coronary CT angiography, which has been used for PHV imaging [2, 3, 7], and 2) a prospectively ECG-triggered protocol. CT parameters are presented in Table 1. Prospective triggering was performed with $128 \times 0.625 \mathrm{~mm}$ collimation and axial imaging with no table movement and $8 \mathrm{~cm}$ exposed at the centre of the gantry. The maximal range that could be reconstructed from these data was $70 \mathrm{~mm}$. No padding was used. Retrospective gating was also performed with $128 \times 0.625 \mathrm{~mm}$ collimation at a pitch of 0.2 . We chose the smallest possible anatomical range $(80.1 \mathrm{~mm})$, which could be covered with the use of this protocol. CT data acquisition only occurred during the $40 \%$ ECG interval (opened PHV) with prospective triggering and reconstructed with retrospective gating. Each valve was imaged 8 times using each protocol, yielding a total of 16 acquisitions per valve. Images were reconstructed at $0.9 \mathrm{~mm}$ thick slices with $0.45 \mathrm{~mm}$ reconstruction increment.

A
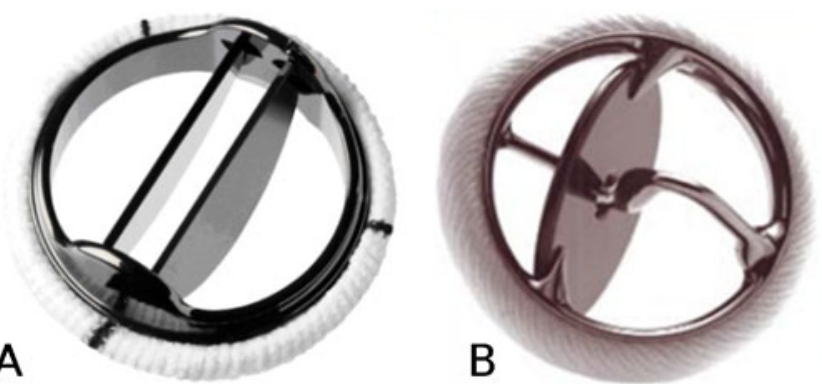

Fig. 1 Photograph of a St Jude (a) and Medtronic Hall aortic valve (b), both pictured from the inflow (ventricular) side. (Images courtesy of St Jude Medical Inc., St Paul, MN, USA, and Medtronic Inc., Minneapolis, MN, USA) 
Fig. 2 The PMMA cylindrical valve chamber in which a mechanical prosthetic valve was tightly mounted (arrow) under a $45^{\circ}$ angle to the gantry (a). Only leaflet motion was possible. The valve chamber was then positioned in a thoracic phantom (b) and connected to the piston pump for leaflet motion
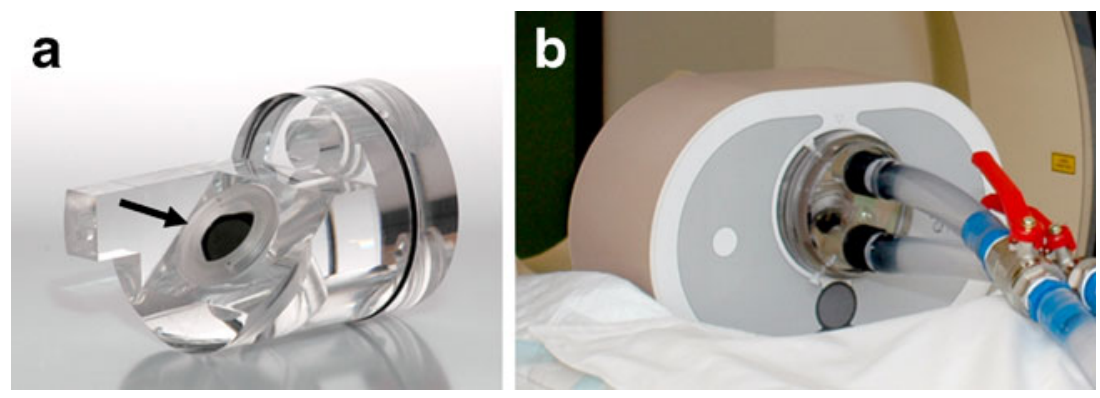

These images were used for HU measurements as well as $3 \mathrm{D}$ volume rendering. Image acquisition with both protocols was performed at 60, 75 and 90 BPM.

All image sets were transferred to a dedicated workstation for analysis (Extended Brilliance Workstation, Philips Medical Systems, Best, The Netherlands). Hyper- and hypodense artefact volumes were quantified in 3D-volume rendered images using two thresholds based on the densities of the surrounding structures according to a methodology described elsewhere for neurosurgical clips [13]. The PMMA in the valve chamber had a density of $130 \pm 15 \mathrm{HU}$, and water $1 \pm 16 \mathrm{HU}$ (mean \pm standard deviation). We chose threshold values that were approximately 3 standard deviations (SD) above the HU of PMMA measurement and below the HU of water, respectively. The chosen thresholds were 175 HU for hyperdense artefacts and -45 HU for hypodense artefacts. Because the PHVs are composed of various radiopaque components such as a titanium ring and tungsten impregnated leaflets, which have CT densities higher than $800 \mathrm{HU}$ [12], the 175-HU threshold included the radiopaque components of the PHV. Hence, the percentage change of the volume measured with the 175-HU threshold underestimates the change in actual artefacts (i.e. the volume measured with the 175-HU threshold without the volume of radiopaque components with densities over $800 \mathrm{HU}$ ) [12]. Areas outside the valve chamber and other unrelated sources of artefacts were digitally excised in an identical manner for all images.

Image noise (defined as the SD of CT attenuation) was measured in all images using a circular region of interest (diameter $1 \mathrm{~cm}$ ) that was placed in an identical section of the PMMA structure of the valve chamber.

\section{Statistical analysis}

Data were analysed using SPSS software (SPSS Statistics Version 16.0, SPSS Inc, Chicago, IL, USA). Non-parametric data were presented as medians with interquartile range (IQR). A Mann-Whitney $U$ test was used for the comparison of artefact volumes and image noise measurements. Statistical significance was defined as $P<0.05$.

\section{Results}

The median artefact volumes for the two thresholds ( -45 and $175 \mathrm{HU})$ at different frequencies are summarised in Table 2. For the St Jude valve, prospective triggering reduced hypodense artefacts (all volume $<-45 \mathrm{HU})$ at $60 \mathrm{BPM}(P=0.001)$, 90 BPM $(P=0.001)$ but not 75 BPM $(P=0.6)$. Hyperdense artefacts (all volume $>175 \mathrm{HU}$ ) were reduced with prospective triggering at $60 \mathrm{BPM}(P=0.001), 75 \mathrm{BPM}(P=0.001)$ and 90 BPM $(P=0.001)$. For the Medtronic Hall valve, hypodense artefacts were reduced with prospective triggering at 60 BPM $(P=0.009), 75$ BPM $(P=0.016)$ and 90 BPM $(P=$ $0.001)$. Hyperdense artefacts were also reduced at all frequencies $(P=0.001$ for 60,75 , and 90 BPM, see Figs. 3 and 4). Volume rendered images illustrate the reduction of artefacts emanating from the leaflet and artefacts related to the prosthetic ring (Fig. 5). The image noise measurements are presented in Table 3. Although significant differences were found between retrospective gating and prospective triggering at 60 and 75 BPM, the image noise did not differ at 90 BPM.

\section{Discussion}

Our findings demonstrate that axial imaging of PHVs using prospective triggering reduces artefacts with much lower

Table 1 CT parameters

\begin{tabular}{lll}
\hline & $\begin{array}{l}\text { Prospective } \\
\text { One ECG interval }\end{array}$ & $\begin{array}{l}\text { Retrospective } \\
\text { All ECG intervals }\end{array}$ \\
\hline Collimation & $128 \times 0.625 \mathrm{~mm}$ & $128 \times 0.625 \mathrm{~mm}$ \\
$\mathrm{kV}$ & 120 & 120 \\
$\mathrm{mAs}$ & 200 & 600 \\
Pitch & 0 & 0.2 \\
Rotation time (ms) & 270 & 270 \\
Filter & Cardiac B & Cardiac B \\
Anatomical length (mm) & 80 & 80.1 \\
CTDI $_{\text {vol }}(\mathrm{mGy})$ & 13.3 & 39.8 \\
DLP & 106.5 & 517.8 \\
\hline
\end{tabular}


Table 2 Artefacts measured in opened (systolic) valves

\begin{tabular}{|c|c|c|c|c|}
\hline \multirow[t]{2}{*}{ Valve } & \multirow[t]{2}{*}{ BPM } & \multirow[t]{2}{*}{ Threshold } & \multicolumn{2}{|c|}{ Median artefact volume $\left(\mathrm{mm}^{3}\right)$ (interquartile range) } \\
\hline & & & Retrospective & Prospective \\
\hline \multirow[t]{6}{*}{ St Jude } & \multirow[t]{2}{*}{60} & $<-45 \mathrm{HU}$ & $2,056(1,939-2,263)$ & $1,168(1,146-1,201)$ \\
\hline & & $>175 \mathrm{HU}$ & $9,000(8,725-10,375)$ & $7,000(6,925-7,150)$ \\
\hline & \multirow[t]{2}{*}{75} & $<-45 \mathrm{HU}$ & $1,609(1,567-1,841)$ & $1,609(1,558-1,641)$ \\
\hline & & $>175 \mathrm{HU}$ & $8,400(7,625-8,775)$ & $6,900(6,700-7,275)$ \\
\hline & \multirow[t]{2}{*}{90} & $<-45 \mathrm{HU}$ & $2,742(1,964-2,896)$ & $1,564(1,527-1,589)$ \\
\hline & & $>175 \mathrm{HU}$ & $9,700(9,100-10,300)$ & $7,000(6,800-7,450)$ \\
\hline \multirow[t]{6}{*}{ Medtronic hall } & \multirow[t]{2}{*}{60} & $<-45 \mathrm{HU}$ & $2,271(1,873-2,630)$ & $1,571(1,474-1,724)$ \\
\hline & & $>175 \mathrm{HU}$ & $8,400(8,300-9,225)$ & $6,150(5,950-6,525)$ \\
\hline & \multirow[t]{2}{*}{75} & $<-45 \mathrm{HU}$ & $2,192(1,788-2,522)$ & $1,608(1,446-1,730)$ \\
\hline & & $>175 \mathrm{HU}$ & $7,550(7,400-8,075)$ & $5,800(5,625-6,075)$ \\
\hline & \multirow[t]{2}{*}{90} & $<-45 \mathrm{HU}$ & $3,164(2,547-3,770)$ & $1,774(1,723-1,977)$ \\
\hline & & $>175 \mathrm{HU}$ & $8,400(7,575-10,000)$ & $5,950(5,725-6,100)$ \\
\hline
\end{tabular}

radiation exposure. In our model, prospective triggering reduced artefact volumes for both valves and at all three frequencies with the exception of hypodense artefacts associated with the St Jude valve at 75 BPM.

The imaging of PHV with MDCT is hampered by artefacts that depend on valve material and valve geometry [1$3,10,12]$. Because of the radiopaque components, these artefacts have been interpreted as metal-related artefacts and efforts to improve image quality have included the increase in tube voltage and current $[3,7]$. Unfortunately, this approach results in sizable dose exposure (and prohibitive exposure for serial evaluations) and therefore limits the usefulness of CT evaluation of PHV. In addition, retrospectively gated acquisition with reconstruction of 10 intervals (i.e. the full cardiac cycle) has been used to offset the variation of the PHV image quality during the cardiac cycle
$[2,3,7]$. Because of the motion of the radiopaque PHV parts, which occurs due to the leaflet excursions and movement of the valve as a whole, reconstructions at each $10 \%$ of the ECG interval may generate only two or three image sets that are of diagnostic quality $[2,11,12]$. In vitro, rapid leaflet motion has been shown to increase hyperdense and hypodense artefacts, and rapid motion of the prosthetic ring, which has a higher density than the leaflets, may be expected to further increase artefacts [12]. In this study, we examined how prospective triggering and retrospective gating affected artefact behaviour and noise in PHV imaging.

In our in vitro model, we tested the effect of prospective triggering at different frequencies. Axial acquisition reduced image noise only for 60 and 75 BPM but not for 90 BPM. This may be explained by differences in the efficiency of dose used for image reconstruction. For example, axial
Fig. 3 Multiplanar reformatted images of the St Jude valve in a view perpendicular to the leaflets acquired with retrospective gating (a) and prospective triggering (b) at 60 BPM. Note the increased deformation of the subprosthetic PMMA contours and increased hyper- and hypodense artefacts in (a)
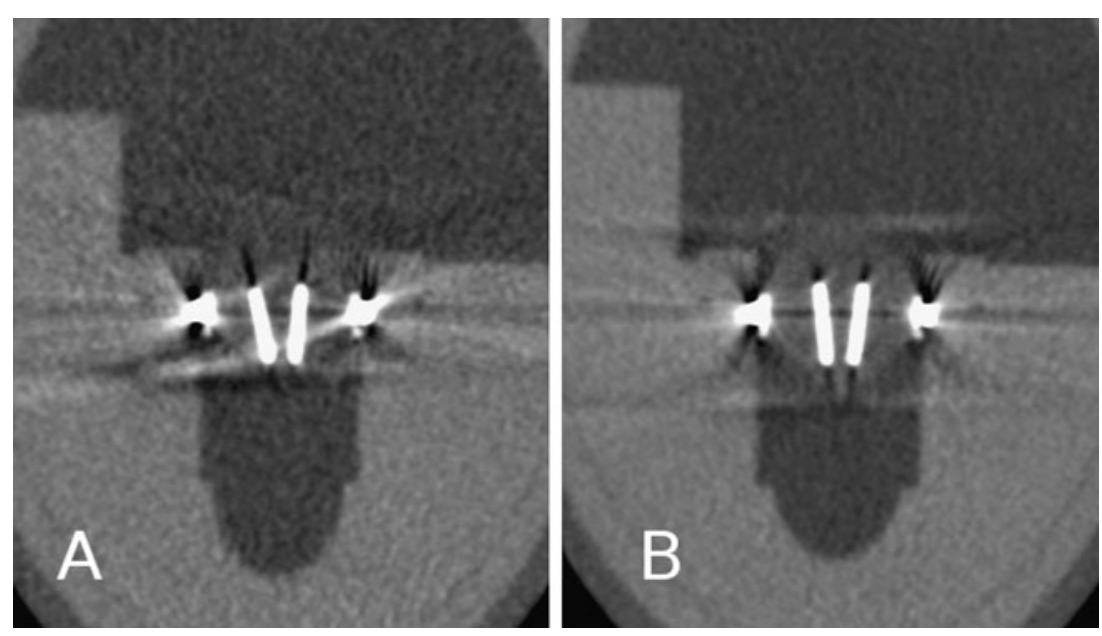
a Artefacts Medtronic Hall: $175 \mathrm{HU}$ threshold

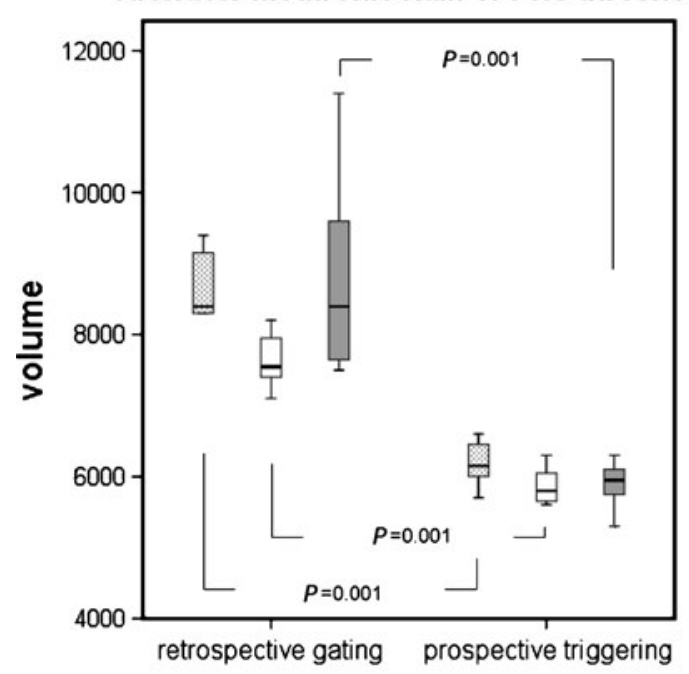

b Artefacts Medtronic Hall: $\mathbf{- 4 5} \mathrm{HU}$ threshold

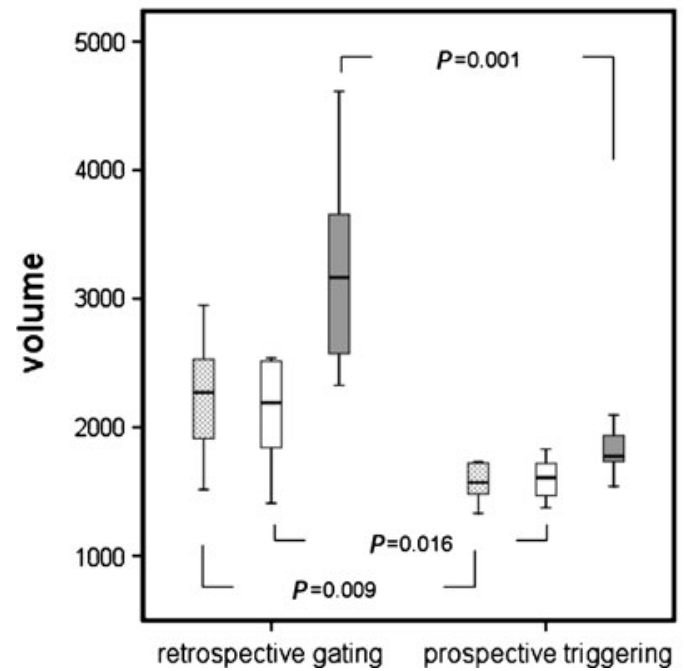

Artefacts St Jude: $175 \mathrm{HU}$ threshold

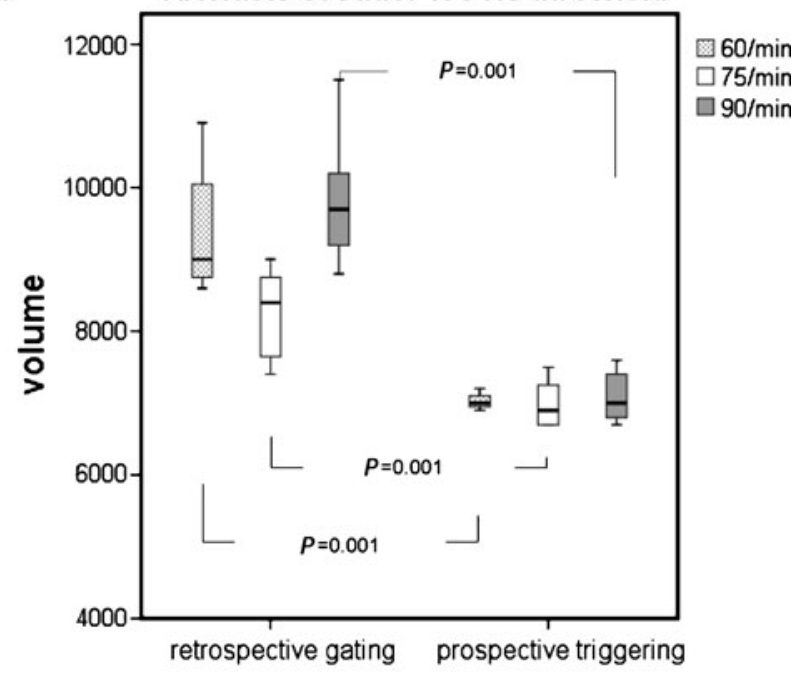

Artefacts St Jude: $-\mathbf{4 5} \mathrm{HU}$ threshold

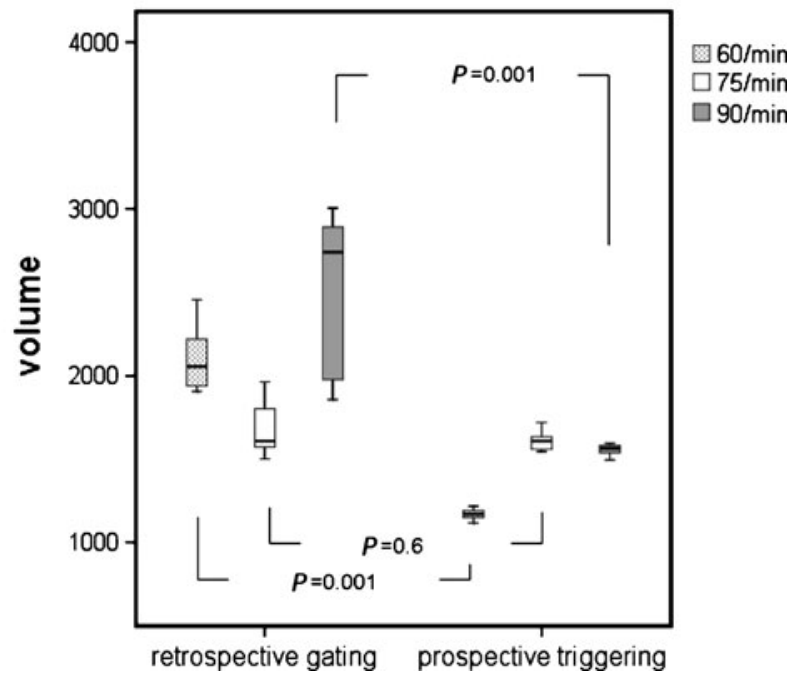

Fig. 4 Changes in a hyperdense and $\mathbf{b}$ hypodense artefacts

acquisition with no padding uses the full dose (200 mAs) for image reconstruction [17]. With equal tube voltage setting, the retrospectively gated protocol at $600 \mathrm{mAs}$ with an R-R interval of $1,000 \mathrm{~ms}$ (frequency $60 / \mathrm{min}$ ) and a nominal temporal resolution of $135 \mathrm{~ms}$ uses only approximately $80 \mathrm{mAs}(=600 \mathrm{mAs} \times 135 / 1,000)$ for the reconstruction of a single ECG interval. At 75 BPM the dose efficiency increases to approximately $100 \mathrm{mAs}$. At 90 BPM, 120 $\mathrm{mAs}$ is used for the reconstruction of a single ECG interval which results in an image noise level comparable to axial acquisition.

Axial acquisition reduced hyperdense and hypodense artefacts except hypodense artefacts of the St Jude valve at 75 BPM. The mechanisms responsible for this reduction are not yet fully understood. The important reduction in hyper- and hypodense artefacts for both valves at 60 and 90 BPM are in contrast to the lack of reduction of hypodense artefacts for the St Jude valve at 75 BPM. Possibly, this seems to be related to lower artefact volumes at 75 BPM with retrospective gating. Also, differences between the two valves may play a role. Earlier in vitro work showed that the opened tilting disc valve led to a sharper increase in hyper- and hypodense artefacts when compared to the St Jude valve [12]. Furthermore, the St Jude valve is composed of a nickel alloy prosthetic ring in contrast to the titanium alloy used for the Medtronic Hall. How these differences would cause a lack of reduction of hypodense artifacts at 75 BPM is not clear. Based on the differences between the two acquisition techniques one may postulate that factors associated with retrospective gating such as multisegment 


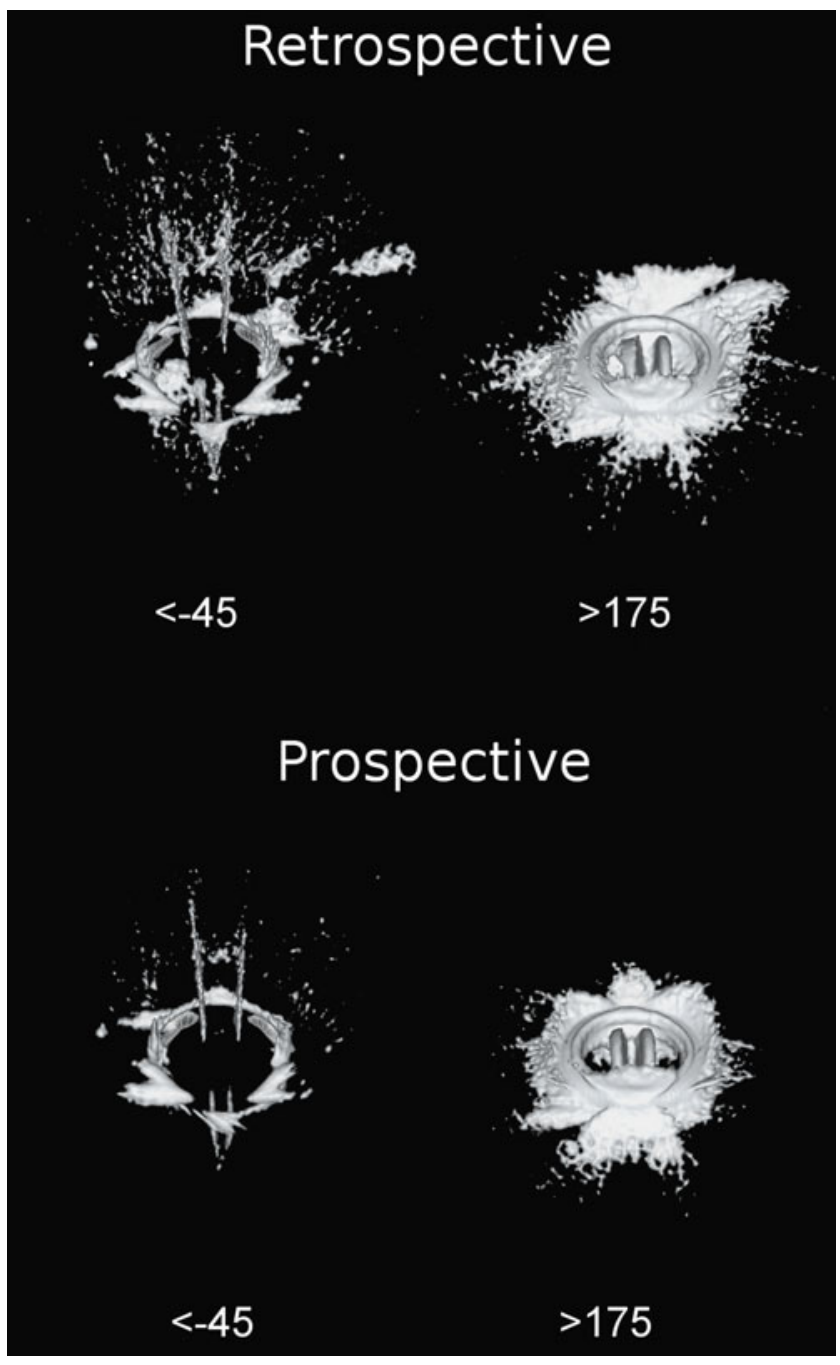

Fig. 5 Volume rendered images of the 3D volume of a St Jude PHV at $60 \mathrm{BPM}$ measured with the $-45 \mathrm{HU}$ threshold (including all volumes less than $-45 \mathrm{HU}$ ) and with the $175 \mathrm{HU}$ threshold (including all volumes with a density higher than $175 \mathrm{HU}$ ). Top panel artefact configurations with retrospective gating. Bottom panel images with prospective triggering. The $175 \mathrm{HU}$ threshold volume includes all radiopaque parts of the valve such as the leaflets and the prosthetic ring

reconstructions and helical interpolation may play a role [1420]. Although increased metal artefacts have been associated with increased noise [14, 15], we found a sharp reduction of hyper- and hypodense artefacts at 90 BPM for both valves despite equal image noise. This suggests that reduction of image noise may not be the primary mechanism responsible for artefacts reduction. However, small variations in leaflet position due to a fluttering motion of the leaflets may cause image discrepancies between consecutive cardiac cycles that increase artefacts with multisegment reconstructions [17, 18]. Furthermore, helical interpolation has been suggested as a mechanism for metal artefacts $[16,18-20]$.

Other experimental work comparing retrospective gating with prospective triggering for metal objects is scarce. Two reports using in vitro models of coronary in-stent restenosis found improved image quality and a reduction in artefacts $[16,20]$. Because of the discrepancy in size and composition of coronary stents, our results are not readily comparable to these reports but similar mechanisms may explain the artefact reduction.

There are several limitations to our study. Prospectively triggered acquisition allows reconstruction of only one preselected cardiac phase and therefore does not allow dynamic imaging. We used only two mechanical prostheses. Possibly, other prostheses consisting of other metal compounds might yield different results. However, the St Jude prosthesis is the most commonly implanted prosthesis worldwide and is the most likely to be encountered clinically. We used the Medtronic Hall valve because it is the most widely implanted tilting disc valve. Both the Medtronic Hall and St Jude valves have been associated with dysfunction due to tissue ingrowth $[2,8,21]$ and for both prostheses $\mathrm{CT}$ imaging has been shown to be of additional value for the diagnosis $[2,4,8]$. Also, we used a phantom in which the valve was fixed and only the leaflets moved. Movement of the valve as a whole may induce more artefacts. Furthermore, the use of different reconstruction filters may change the amount of PHV-induced artefacts.

Our results suggest a reduction of most PHV-related artefacts at different frequencies that appears independent of the image noise reduction with axial acquisition. Prerequisites, however, are a regular heart rate and the correct timing relative to the cardiac cycle.
Table 3 Image noise (median and interquartile range) for retrospective gating and prospective triggering a The $P$ values result from Mann-Whitney $U$ test

\begin{tabular}{lllll}
\hline Valve & Beats per minute & Retrospective gating & Prospective triggering & $P$ values $^{\mathrm{a}}$ \\
\hline St Jude & 60 & $16.0(14.5-17.0)$ & $11.0(10.3-12.0)$ & 0.001 \\
& 75 & $18.0(17.0-18.8)$ & $13.5(12.3-14.8)$ & 0.001 \\
\multirow{3}{*}{ Medtronic hall } & 60 & $14.0(12.3-16.0)$ & $13.0(12.3-13.8)$ & 0.198 \\
& 75 & $16.0(14.3-17.0)$ & $11.0(10.3-11.8)$ & 0.001 \\
& 90 & $17.5(15.0-19.8)$ & $14.0(12.5-16.0)$ & 0.026 \\
& $15.0(14.3-15.8)$ & $14.5(13.0-15.0)$ & 0.269 \\
\hline
\end{tabular}


Acknowledgements This study was supported by a grant from the Netherlands Heart Foundation [Grant number 2009B014]. We thank Aron de Vries and Jacob Gebreegziabeher for their help with the image analysis.

Open Access This article is distributed under the terms of the Creative Commons Attribution Noncommercial License which permits any noncommercial use, distribution, and reproduction in any medium, provided the original author(s) and source are credited.

\section{References}

1. Tsai IC, Lin YK, Chang Y, Fu YC, Wang CC, Hsieh SR, Wei HJ, Tsai HW, Jan SL, Wang KY, Chen MC, Chen CC (2009) Correctness of multi-detector-row computed tomography for diagnosing mechanical prosthetic heart valve disorders using operative findings as a gold standard. Eur Radiol 19:857-867

2. Symersky P, Budde RPJ, de Mol BAJM, Prokop M (2009) Comparison of multidetector-row computed tomography to echocardiography and fluoroscopy for evaluation of patients with mechanical prosthetic valve obstruction. Am J Cardiol 104:1128-1134

3. Konen E, Goitein O, Feinberg MS, Eshet Y, Raanani E, Rimon U, Di-Segni E (2008) The role of ECG-gated MDCT in the evaluation of aortic and mitral mechanical valves: initial experience. Am J Roentgenol 191:26-31

4. Habets J, Budde RP, Symersky P, van den Brink RB, de Mol BA, Mali WP, van Herwerden LA, Chamuleau SA (2011) Diagnostic evaluation of left-sided prosthetic heart dysfunction. Nat Rev Cardiol 8:466-478

5. Girard SE, Miller FA, Orszulak TA, Mullany CJ, Montgomery S, Edwards WD, Tazelaar HD, Malouf JF, Tajik AJ (2001) Reoperation for prosthetic aortic valve obstruction in the era of echocardiography: trends in diagnostic testing and comparison with surgical findings. J Am Coll Cardiol 37:579-584

6. Faletra F, Constantin C, De Chiara F, Masciocco G, Santambrogio G, Moreo A, Alberti A, Vitali E, Pellegrini A (2000) Incorrect echocardiographic diagnosis in patients with mechanical prosthetic valve dysfunction: correlation with surgical findings. Am J Med 108:531-537

7. Chenot F, Montant P, Goffinet C, Pasquet A, Vancraeynest D, Coche E, Vanoverschelde JL, Gerber BL (2010) Evaluation of anatomic valve opening and leaflet morphology in aortic valve bioprosthesis by using multidetector CT: comparison with transthoracic echocardiography. Radiology 255:377-385
8. Teshima H, Hayashida N, Fukunaga S, Tayama E, Kawara T, Aoyagi S, Uchida M (2004) Usefulness of a multidetector-row computed tomography scanner for detecting pannus formation. Ann Thorac Surg 77:523-526

9. Toledano D, Acar C (2010) Usefulness of computed tomography scanning in the diagnosis of aortic prosthetic valve pannus. J Heart Valve Dis 19:665-668

10. Symersky P, Budde RPJ, Prokop M, de Mol BA (2011) Multidetector-row computed tomography imaging characteristics of mechanical prosthetic valves. J Heart Valve Dis 20:216-222

11. Habets J, Symersky P, van Herwerden LA, de Mol BA, Spijkerboer AM, Mali WP, Budde RP (2011) Prosthetic heart valve assessment with multidetector-row CT; imaging characteristics of 91 valves in 83 patients. Eur Radiol 21:1390-1396

12. Symersky P, Budde RPJ, Westers P, de Mol BAJM, Prokop M (2011) Multidetector CT imaging of mechanical prosthetic heart valves: quantification of artifacts with a pulsatile in-vitro model. Eur Radiol 21:2103-2110

13. van der Schaaf I, van Leeuwen M, Vlassenbroek A, Velthuis B (2006) Minimizing clip artifacts in multi CT angiography in clipped patients. Am J Neuroradiol 27:60-66

14. Boas FE, Fleischmann D (2011) Evaluation of two iterative techniques for reducing metal artifacts in computed tomography. Radiology 259:894-902

15. De Man B, Nuyts J, Dupont P, Marchal G, Suetens P (1999) Metal streak artifacts in $\mathrm{X}$-ray computed tomography: a simulation study. IEEE Trans Nucl Sci 46:691-696

16. Horiguchi J, Fujioka C, Kiguchi M, Yamamoto H, Kitagawa T, Kohno S, Ito K (2009) Prospective ECG-triggered axial CT at 140$\mathrm{kV}$ tube voltage improves coronary in-stent restenosis at a lower radiation dose compared with conventional retrospective ECGgated helical CT. Eur Radiol 19:2363-2372

17. Prokop M (2003) Principles of CT, spiral CT and multislice CT. In: Prokop M, Galanski M (eds) Spiral and multislice computed tomography of the body. Thieme Verlag, New York, pp 1-43

18. Barrett JF, Keat N (2004) Artifacts in CT: recognition and avoidance. Radiographics 24:1679-1691

19. Wilting JE, Timmer J (1999) Artefacts in spiral-CT images and their relation to pitch and subject morphology. Eur Radiol 9:316-322

20. Yang WJ, Pan ZL, Zhang H, Pang LF, Guo Y, Chen KM (2011) Evaluation of coronary artery in-stent restenosis with prospectively ECG-triggered axial CT angiography versus retrospective technique: a phantom study. Radiol Med 116:189-196

21. Cho YH, Jeong DS, Park PW, Park KH, Sung K, Kim WS, Lee YT (2011) Serial changes of hemodynamic performance with Medtronic Hall valve in the aortic position. Ann Thor Surg 91:424-431 